\title{
Outcomes of empiric treatment for pediatric tuberculosis, Kampala, Uganda, 2010-2015
}

\author{
Eric Wobudeya ${ }^{1{ }^{*}+}$ (D), Devan Jaganath ${ }^{2 \dagger}$, Moorine Penninah Sekadde ${ }^{3}$, Betty Nsangi ${ }^{4}$, Heather Haq ${ }^{5}$ and
} Adithya Cattamanchi $i^{6,7,8}$

\begin{abstract}
Background: Childhood tuberculosis (TB) diagnoses often lack microbiologic confirmation and require empiric treatment. Barriers to empiric treatment include concern for poor outcomes and adverse effects. We thus determined the outcomes of empiric TB treatment from a retrospective cohort of children at a national referral hospital in Kampala, Uganda from 2010 to 2015.

Methods: Children were diagnosed clinically and followed through treatment. Demographics, clinical data, outcome and any adverse events were extracted from patient charts. A favorable outcome was defined as a child completing treatment with clinical improvement. We performed logistic regression to assess factors associated with loss to follow up and death.

Results: Of 516 children, median age was 36 months (IQR 15-73), 55\% (95\% CI 51-60\%) were male, and HIV prevalence was $6 \%$ (95\% Cl 4-9\%). The majority $(n=422,82,95 \% \mathrm{Cl} 78-85 \%)$ had a favorable outcome, with no adverse events that required treatment discontinuation. The most common unfavorable outcomes were loss to follow-up $(57 / 94,61 \%)$ and death (35/94, 37\%; overall mortality 7\%). In regression analysis, loss to follow up was associated with age 10-14 years (OR 2.38, 95\% Cl 1.15-4.93, $p=0.02$ ), HIV positivity (OR 3.35, 95\% Cl 1.41-7.92, $p=0.01$ ), hospitalization (OR 4.14,95\% Cl 2.08-8.25, $p<0.001$ ), and living outside of Kampala (OR 2.64, 95\% Cl $1.47-4.71, p=0.001)$. Death was associated with hospitalization (OR 4.57, 95\% Cl 2.0-10.46, $p<0.001$ ), severe malnutrition (OR 2.98, 95\% Cl 1.07-8.27, $p=0.04)$, baseline hepatomegaly (OR 4.11, 95\% Cl 2.09-8.09, $p<0.001$ ), and living outside of Kampala (OR 2.41, 95\% Cl 1.17-4.96, $p=0.02$ ).
\end{abstract}

Conclusions: Empiric treatment of child TB was effective and safe, but treatment success remained below the $90 \%$ target. Addressing co-morbidities and improving retention in care may reduce unfavorable outcomes.

Keywords: Child, Tuberculosis, Treatment, Outcomes

\section{Background}

Timely initiation of treatment is critical for effective tuberculosis $(\mathrm{TB})$ care and control in children. Of the estimated 233,000 children that die from TB each year, $96 \%$ did not receive treatment $[1,2]$. For primary care providers in $\mathrm{TB}$ endemic settings, challenges with confirming a TB diagnosis in children and concern of

\footnotetext{
* Correspondence: ewobudeya@gmail.com

tEric Wobudeya and Devan Jaganath contributed equally to this work. 1 Directorate of Pediatrics \& Child Health, Mulago National Referral Hospital, P.O. Box 23491, Kampala, Uganda

Full list of author information is available at the end of the article
}

adverse effects with empiric treatment (i.e., without microbiological confirmation) are key barriers to initiating anti-TB treatment $[3,4]$. Symptoms are non-specific, chest $\mathrm{x}$-ray findings variable, and diagnostic testing is often unavailable or not feasible due to difficulty obtaining sputum $[3,5]$. Even when diagnostic testing occurs, sensitivity is decreased due to the paucibacillary nature of pediatric TB [6]. This contributes to $55 \%$ of child TB cases not being reported to national programs [2] from high TB burden settings have consistently documented that health care workers feel uncomfortable about making a TB diagnosis in a child and that there are often

(c) The Author(s). 2019 Open Access This article is distributed under the terms of the Creative Commons Attribution 4.0 International License (http://creativecommons.org/licenses/by/4.0/), which permits unrestricted use, distribution, and reproduction in any medium, provided you give appropriate credit to the original author(s) and the source, provide a link to the Creative Commons license, and indicate if changes were made. The Creative Commons Public Domain Dedication waiver (http://creativecommons.org/publicdomain/zero/1.0/) applies to the data made available in this article, unless otherwise stated. 
delays in care [7-9]. For example, a cross-sectional study of six primary clinics in Kampala found that no children who met clinical criteria for TB had been started on anti-TB treatment [10]. While algorithms exist to identify children with TB based on clinical factors [11-13], a concern is that without microbiologic confirmation, misdiagnosis could result in poor outcomes or adverse events from anti-TB treatment. A better understanding of outcomes of children treated empirically for TB based on a clinical diagnosis would provide more guidance to providers about the effectiveness and safety. We thus analyzed outcomes of children less than 15 years of age with clinically diagnosed $\mathrm{TB}$ receiving empiric treatment over a five-year period at a national referral hospital in Kampala, Uganda, and factors associated with the unfavorable outcomes of lost to follow up and death.

\section{Methods}

\section{Study design and setting}

This was a retrospective cohort study of children treated for clinically diagnosed TB at Mulago National Referral Hospital Pediatric TB Clinic. The clinic treats children up to 14 years of age with drug-susceptible TB using Fixed Dose Combination (FDC) treatment in accordance with Uganda National TB and Leprosy Programme (NTLP) guidelines [13]. Children are seen in clinic every two weeks during the first month of treatment and monthly thereafter for the standard six-month or twelve-month treatment depending on the TB disease type. At each visit, clinical notes are documented using standardized forms and entered into a secure electronic database. Adverse events are monitored based on clinical symptoms and exam, without routine laboratory testing. Management of any adverse events were completed according to national guidelines [12]. The Mulago Hospital Research and Ethics Committee approved the study protocol and waived the requirement for informed consent.

\section{Study population}

We included all children less than 15 years old treated empirically for TB between January 2010 and December 2015. Clinical diagnosis was made per NTLP guidelines $[12,13]$. We excluded children if they had multi-drug resistant (MDR) TB, were transferred, or were missing key variables (age and treatment outcome). As a comparison, outcomes on children with confirmed TB were also collected.

\section{Data collection and definitions}

The data was not publically available hence we obtained Mulago Hospital permission to use the data. We extracted demographic and clinical data including HIV status, type of $\mathrm{TB}, \mathrm{TB}$ treatment outcome, and any adverse events from the Pediatric TB Clinic electronic database. We defined severe malnutrition as a weightfor-age $\mathrm{Z}$ score less than -3 . We defined a favorable outcome if the child completed treatment and had documented clinical improvement, and an unfavorable outcome if the child died, failed treatment (no clinical improvement or treatment discontinuation) or was lost to follow-up prior to completion of treatment.

\section{Statistical analysis}

Descriptive statistics were assessed with proportions and 95\% confidence intervals (CI) for categorical variables and median and interquartile range (IQR) for continuous variables. For bivariate analysis of characteristics associated with favorable versus unfavorable outcome, we compared proportions using the chi-squared test. We stratified unfavorable outcomes into loss to follow up and death, and conducted logistic regression on characteristics with $p$-value $<0.05$ from the unfavorable bivariate analysis. HIV status and severe malnutrition (known to be associated with unfavorable outcome) $[14,15]$, and sex were also included. Odds Ratios (OR) were presented with 95\% Confidence Intervals (CI); $p$-value $<0.05$ was considered significant. We performed analyses using STATA 15 (Stata Corp, College Station, TX, USA).

\section{Results}

\section{Cohort characteristics}

Of 713 children treated for TB during the study period, there were 64 with confirmed TB, 1 with MDR TB, 1 with missing age information, 23 who were transferred out, and 108 with missing treatment outcome (Fig. 1), for a total of 516 children empirically started on anti-TB treatment.

Details of patient characteristics by outcome are shown in Table 1 . The median age was 36 months (IQR 15-73), 55\% (95\% CI 51-60\%) were male, and about half resided outside of Kampala district (46, 95\% CI 41$50 \%)$. The majority $(65,95 \%$ CI $61-69 \%)$ were below five years old, and HIV prevalence was 6\% (31/509, 95\% CI 4-9\%). The prevalence of severe malnutrition was $22 \%(76 / 349,95 \%$ CI $18-26 \%)$. Over two-thirds $(69 \%, 354 / 515,95 \%$ CI $65-73 \%)$ of diagnoses were pulmonary TB cases.

\section{Outcomes of empiric treatment}

Of the 516 children empirically started on anti-TB treatment, $422(82 \%)$ children had a favorable treatment outcome. Of the 94 children with unfavorable outcomes, $61 \%$ (57/94) were lost to follow-up, 37\% (35/94) died, and $2 \%(2 / 94)$ failed treatment. The majority of deaths were in children under five years $(22 / 35,63 \%)$; three deaths occurred in children infected with HIV. The 


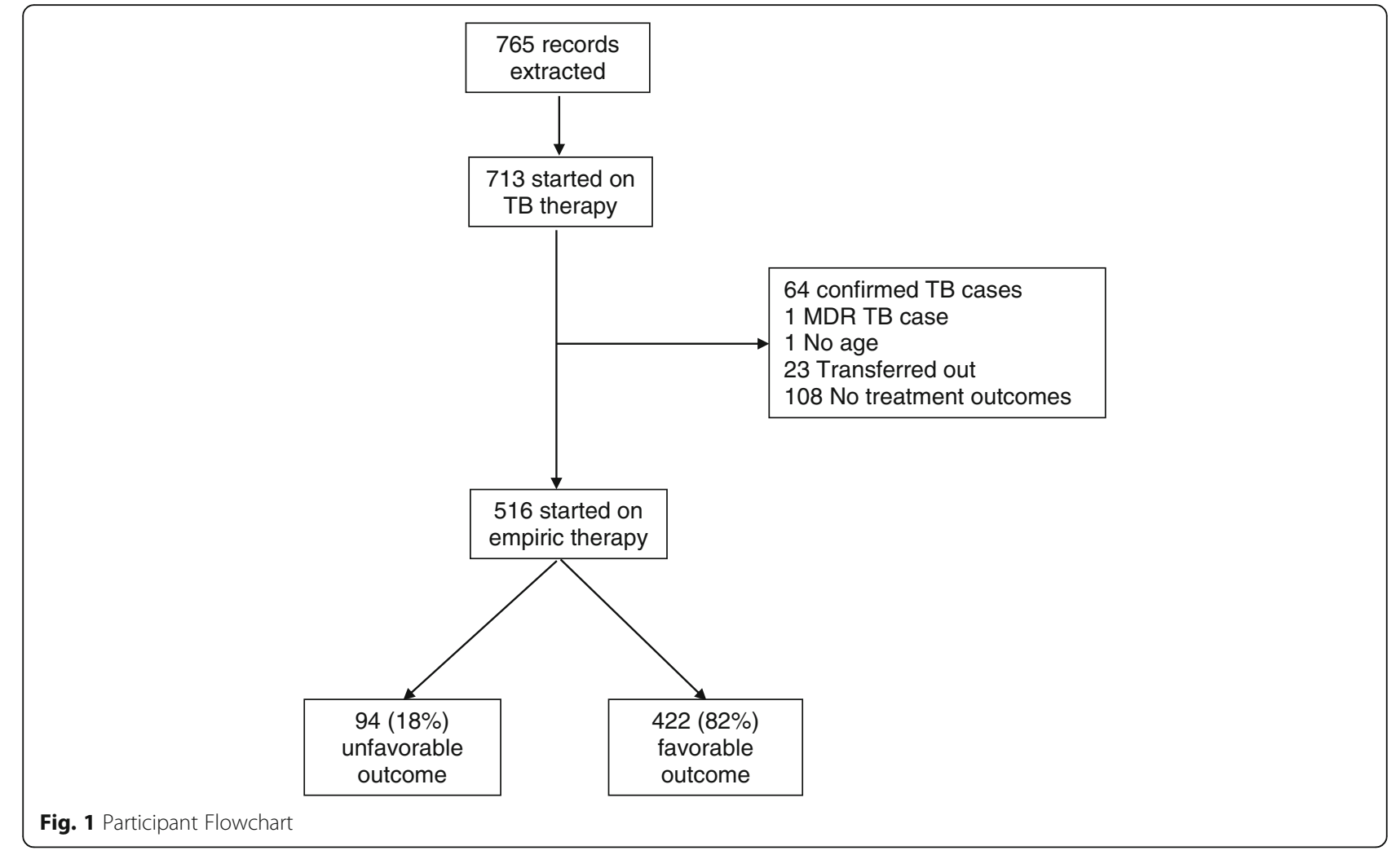

Table 1 Demographic and clinical characteristics of children empirically treated for TB at Mulago Pediatric TB unit $(N=516)^{*}$

\begin{tabular}{|c|c|c|c|}
\hline \multirow[t]{2}{*}{ Characteristic } & Favorable $(N=422)$ & Unfavorable $(N=94)$ & \multirow[t]{2}{*}{$p$-value } \\
\hline & $\mathrm{N}(\%, 95 \mathrm{Cl})$ & $\mathrm{N}(\%, 95 \mathrm{Cl})$ & \\
\hline \multicolumn{4}{|l|}{ Age group } \\
\hline$<5$ years & $280(66,62-71)$ & $57(61,50-70)$ & \multirow[t]{3}{*}{0.006} \\
\hline $5-9$ years & $104(25,21-29)$ & $18(19,12-28)$ & \\
\hline 10-14 years & $38(9,7-12)$ & $19(20,13-30)$ & \\
\hline Male Sex & $238(56,52-61)$ & $48(51,41-61)$ & 0.35 \\
\hline Reside outside of Kampala & $174(41,37-46)$ & $62(66,56-75)$ & $<0.001$ \\
\hline Pulmonary TB $(n=515)$ & $291(69,65-73)$ & $53(67,57-76)$ & 0.69 \\
\hline HIV positive $(n=509)$ & $19(5,3-7)$ & $12(14,8-23)$ & 0.001 \\
\hline Severe Malnutrition ${ }^{\ddagger}(n=349)$ & $60(20,16-25)$ & $16(32,20-46)$ & 0.06 \\
\hline Hospitalized $(n=480)$ & $33(8,6-11)$ & $26(34,24-45)$ & $<0.001$ \\
\hline Abnormal Chest X-ray $(n=367)$ & $297(93,90-96)$ & $46(94,82-98)$ & 1.0 \\
\hline BCG vaccinated $(n=301)$ & $239(92,88-95)$ & $35(83,68-92)$ & 0.06 \\
\hline Baseline Hepatomegaly ( $n=383$ ) & $28(8,6-12)$ & $13(30,18-46)$ & $<0.001$ \\
\hline TST Positive $(n=246)$ & $161(75,69-80)$ & $18(58,40-74)$ & 0.05 \\
\hline
\end{tabular}

${ }^{*} \mathrm{~N}=516$ unless missing data, with number available indicated in parentheses $\dagger p$-value by Chi-squared or Fisher's exact testing

₹ Severe malnutrition defined as weight-for-age $Z$ score less than -3 
proportion of children on empiric treatment with favorable outcomes was similar to the children who had confirmed TB (48 of 54 with outcomes, $89 \%$, versus $82 \%$ on empiric treatment, $p=0.17$ ).

In total, there were eight drug reactions reported at follow up visits, with complaints that included paresthesia in the lower limbs, scaling in the plantar foot, vomiting, pallor and myalgia. However, there were no adverse events reported that required treatment discontinuation.

Unfavorable outcomes were more likely among children aged 10-14 years, HIV positive, not residing in Kampala, with baseline hepatomegaly, and hospitalization (Table 1). When stratified by loss to follow up and death (Table 2), loss to follow up was associated with age 10-14 years (OR 2.38, 95\% CI 1.154.93, $p=0.02$ ), HIV infection (OR 3.35, 95\% CI 1.417.92, $p=0.01$ ), hospitalization (OR 4.14, 95\% CI 2.08$8.25, p<0.001$ ), and residing outside of Kampala (OR $2.64,95 \%$ CI $1.47-4.71, p=0.001)$. Death was associated with being hospitalized (OR 4.57, 95\% CI 2.010.46, $p<0.001$ ), severe malnutrition (OR 2.98, 95\% CI $1.07-8.27, p=0.04$ ), hepatomegaly at baseline (OR 4.11, 95\% CI 2.09-8.09, $\mathrm{p}<0.001$ ), and residing outside of Kampala (OR 2.41, 95\% CI 1.17-4.96, $p=0.02$ ).

\section{Discussion}

In this study, we describe empiric treatment outcomes of children clinically diagnosed with TB at a referral center in Kampala, Uganda from 2010 to 2015. The majority of children $(82 \%)$ had a favorable outcome with clinical improvement. However, this is below the World Health Organization (WHO) target of $90 \%$ treatment success [16]. There were no adverse events that required discontinuation of treatment. Loss to follow-up was the most common unfavorable outcome and was associated with older age (10-14 years), HIV infection, hospitalization, and living outside of Kampala. Death was the next cause of unfavorable outcome, and was associated with hospitalization, severe malnutrition, baseline hepatomegaly, and living outside of Kampala. Thus, empiric treatment was found to be safe and effective for most children, but greater efforts are needed to improve outcomes.

While the proportion with favorable outcome was below the global target, it corresponds with child outcomes in similar settings [16]. Retrospective studies in Nigeria, South Africa and Ethiopia found child TB (confirmed and clinically diagnosed) success rates of $77.4 \%$ [17], $78 \%$ [18], and $85.5 \%[19,20]$, respectively. We also found a similar favorable proportion among the confirmed child TB cases. Our data support that empiric treatment of $\mathrm{TB}$ in children without microbiologic confirmation does not lead to worse outcomes.

Loss to follow-up was the most common reason for an unfavorable outcome. HIV co-infection has been associated with loss to follow up in youth [21,22], and may be related to the additional pill burden, stigma and fear of discrimination. Hospitalization and not living in Kampala also were associated with loss to follow up; improved efforts are needed to ensure follow up after a child is discharged from the hospital, and to address barriers to obtaining care if the child does not live near the clinic. For example, Defeat TB is an initiative in Uganda to improve TB treatment success through health system strengthening to improve coordination of care and decentralize TB diagnosis and management [23].

Children age 10-14 years were more than twice as likely to be lost to follow up as children under 5 years. Compared to younger children, adolescents with TB face unique challenges to their care, including greater peer pressure, stigma, behavioral issues, substance abuse and prevalence of co-morbidities including HIV [2]. A retrospective analysis in South Africa found that $15 \%$ of adolescents with HIV aged 15-19years discontinued TB

Table 2 Factors associated with loss to follow up or death in children empirically treated at Mulago Pediatric TB unit, 2010-2015

\begin{tabular}{|c|c|c|c|c|}
\hline & \multicolumn{2}{|c|}{ Loss to Follow Up } & \multicolumn{2}{|l|}{ Death } \\
\hline & $\mathrm{OR}(95 \% \mathrm{Cl})$ & $\overline{p \text {-value }}$ & $\mathrm{OR}(95 \% \mathrm{Cl})$ & $p$-value \\
\hline \multicolumn{5}{|l|}{ Age Group } \\
\hline$<5$ yrs & REF & - & REF & - \\
\hline $5-9 \mathrm{yrs}$ & $0.88(0.43-1.80)$ & 0.73 & $0.87(0.36-2.09)$ & 0.76 \\
\hline $10-14$ yrs & $2.38(1.15-4.93)$ & 0.02 & $1.68(0.65-4.36)$ & 0.28 \\
\hline Male sex & $0.75(0.43-1.31)$ & 0.31 & $0.84(0.42-1.67)$ & 0.62 \\
\hline HIV positive & $3.35(1.41-7.92)$ & 0.01 & $1.60(0.46-5.57)$ & 0.46 \\
\hline Hospitalization & $4.14(2.08-8.25)$ & $<0.001$ & $4.57(2.0-10.46)$ & $<0.001$ \\
\hline resides outside of Kampala & $2.64(1.47-4.71)$ & 0.001 & $2.41(1.17-4.96)$ & 0.02 \\
\hline Severe Malnutrition & $1.46(0.65-3.3)$ & 0.36 & $2.98(1.07-8.27)$ & 0.04 \\
\hline Baseline Hepatomegaly & $1.36(0.83-2.22)$ & 0.22 & $4.11(2.09-8.09)$ & $<0.001$ \\
\hline
\end{tabular}

Cl Confidence Interval, OR odds ratio, HIV Human Immunodeficiency Virus 
treatment [24]. A study in Botswana found that adolescents were twice as likely to be lost to follow-up compared to adult [21]. These results emphasize that adolescent-friendly TB programs are needed to address their unique issues and continue to engage adolescents in care.

Mortality was the second most common unfavorable outcome. Our overall mortality was high at $7 \%$, similar to the study in Nigeria that found a child TB mortality of 6\% [17]. Consistent with past studies [17-19], our analyses found that factors related to more severe disease were associated with death, namely hospitalization, severe malnutrition and hepatomegaly. Living outside of Kampala district was also associated with death, and may reflect socioeconomic factors or delays in diagnosis and management.

Prior assessments of child TB outcomes have included both confirmed and clinically diagnosed TB. Confirmed cases may reflect children with higher bacterial load or occur in settings where there is greater access to diagnostics. By focusing only on children empirically treated for TB based on a clinical diagnosis, we sought to provide outcome data that was more generalizable to the majority of health care workers in low-resource settings.

There were also some potential limitations to our study. As a retrospective cohort, we cannot comment on the accuracy of the clinical diagnoses, although documented symptoms and signs were consistent with national guidelines. The study was conducted at a referral hospital, and may lower generalizability to primary care clinics. Mortality from alternative diagnoses were possible, and the exact causes of death were unknown. Adherence was not consistently documented. There was no available data on contacts with multi-drug resistant (MDR) TB to address empiric MDR treatment. The sample had a low proportion with HIV infection and limits the assessment of outcomes of empiric TB treatment in HIV-TB co-infected children. In particular, HIV-related mortality was low, and may be an underestimate as we did not include children with confirmed TB, who were transferred out or who had missing outcomes. The low number of unfavorable outcomes did not provide the power for a multivariable stratified regression analysis. Data was also not available on any follow-up laboratory testing to determine sub-clinical adverse events such as elevated liver transaminases on treatment.

\section{Conclusions}

Our results highlight that initiation of empiric TB care in children is overall safe and effective if health care workers use appropriate clinical guidelines. However, they also suggest that diagnosis and initiation of treatment are only the beginning of the care cascade; health facility strengthening and age-appropriate care is critical to ensure favorable outcomes and retention in care.

\section{Abbreviations}

aOR: Adjusted odds ratio; BCG: Bacillus Calmette-Guérin; Cl: Confidence interval; FDC: Fixed Dose Combination; HIV: Human Immunodeficiency Virus; IQR: Interquartile Range; MDR: Multi-drug resistant; OR: Odds ratio;

TB: Tuberculosis; TST: Tuberculin Skin Test; WHO: World Health Organization

\section{Acknowledgements}

We thank the families, staff, and administration at the Mulago National Referral Hospital for their efforts in diagnosing and managing these children with TB.

\section{Funding \\ DJ is a Fellow in the Pediatric Scientist Development Program. This project was supported by grants from the Eunice Kennedy Shriver National Institute of Child Health and Human Development (K12 HD000850) and the National Heart, Lung, and Blood Institute (R01 HL139717). The funders had no role in the design, collection, analysis, interpretation, or writing of the study.}

\section{Availability of data and materials}

The datasets used and/or analyzed during the current study are available from the corresponding author on reasonable request.

\section{Authors' contributions}

DJ, EW and AC conceptualized the study and analysis. EW, MPS, BN and HH provided oversight and collection of the data. EW and DJ conducted the analysis. DJ and EW drafted the manuscript, and all authors provided revisions. All authors have read and approved the manuscript.

\section{Ethics approval and consent to participate}

The Mulago Hospital Research and Ethics Committee approved the study protocol and waived the requirement for informed consent. We conduct chart reviews and did not get into contact with human subjects.

\section{Consent for publication}

Not applicable.

\section{Competing interests}

EW is an associate editor of the BMC Public Health journal. All the authors declare that they have no competing interests.

\section{Publisher's Note}

Springer Nature remains neutral with regard to jurisdictional claims in published maps and institutional affiliations.

\section{Author details}

${ }^{1}$ Directorate of Pediatrics \& Child Health, Mulago National Referral Hospital, P.O. Box 23491, Kampala, Uganda. 'Division of Pediatric Infectious Diseases, University of California, 550 16th St. 4th floor, San Francisco, CA 94158, USA. ${ }^{3}$ National TB and Leprosy Program (NTLP), Plot 6, Lourdel Road, Nakasero, P. O. Box 7272, Kampala, Uganda. ${ }^{4}$ USAID RHITES-EC, University Research Co. LLC, Plot 40, Ntinda II Road, PO Box 28745, Kampala, Uganda. ${ }^{5}$ Department of Pediatrics, Baylor College of Medicine, Houston, TX, USA. ${ }^{6}$ Division of Pulmonary and Critical Care Medicine, University of California, 1001 Potrero Ave, SFGH 5, San Francisco, CA 94110, USA. ${ }^{7}$ Center for Vulnerable Populations, Department of Medicine, University of California, San Francisco, USA. ${ }^{8}$ Curry International Tuberculosis Center, University of California, San Francisco, USA.

Received: 7 February 2019 Accepted: 15 April 2019

Published online: 29 April 2019

\section{References}

1. Dodd PJ, Yuen CM, Sismanidis C, Seddon JA, Jenkins HE. The global burden of tuberculosis mortality in children: a mathematical modelling study. Lancet Glob Health. 2017;5(9):e898-906.

2. World Health Organization. Roadmap towards ending TB in children and adolescents.10 Dec 2018. Available from: https://www.who.int/tb/ publications/2018/tb-childhoodroadmap/en/.

3. Marais BJ. Improving access to tuberculosis preventive therapy and treatment for children. Int J Infect Dis. 2017;56:122-5. 
4. Chiang SS, Roche S, Contreras C, Alarcon V, Del Castillo H, Becerra MC, et al. Barriers to the diagnosis of childhood tuberculosis: a qualitative study. Int J Tuberc Lung Dis. 2015;19(10):1144-52.

5. Reid MJ, Saito S, Fayorsey R, Carter RJ, Abrams EJ. Assessing capacity for diagnosing tuberculosis in children in sub-Saharan African HIV care settings. Int J Tuberc Lung Dis. 2012;16(7):924-7.

6. Dunn JJ, Starke JR, Revell PA. Laboratory diagnosis of mycobacterium tuberculosis infection and disease in children. J Clin Microbiol. 2016;54(6):1434-41.

7. Arscott-Mills T, Masole L, Ncube R, Steenhoff AP. Survey of health care worker knowledge about childhood tuberculosis in high-burden centers in Botswana. Int J Tuberc Lung Dis. 2017;21(5):586-91.

8. Szkwarko D, Hirsch-Moverman Y, Du Plessis L, Du Preez K, Carr C, Mandalakas AM. Child contact management in high tuberculosis burden countries: a mixed-methods systematic review. PLoS One. 2017;12(8):e0182185.

9. Sullivan BJ, Esmaili BE, Cunningham CK. Barriers to initiating tuberculosis treatment in sub-Saharan Africa: a systematic review focused on children and youth. Glob Health Action. 2017;10(1):1290317.

10. Kizito S, Katamba A, Marquez C, Turimumahoro P, Ayakaka I, Davis JL, et al. Quality of care in childhood tuberculosis diagnosis at primary care clinics in Kampala, Uganda. Int J Tuberc Lung Dis. 2018;22(10):1196-202.

11. International Union Against Tuberculosis and Lung Disease (The Union). Desk-guide for diagnosis and management of TB in children. Paris, France: The Union; 2010.

12. Uganda National Tuberculosis and Leprosy Programme. Management of Tuberculosis in Children: A Health Worker Guide. 2015; 10 Dec 2018 Available from: http://health.go.ug/download/file/fid/1536.

13. Uganda National TB and Leprosy Programme. Manual for management and control of Tuberculosis and Leprosy in Uganda, 3rd Edition. Kampala, Uganda. 2017. Available from: http://health.go.ug/download/file/fid/1539.

14. Swaminathan S, Rekha B. Pediatric tuberculosis: global overview and challenges. Clin Infect Dis. 2010;50(Suppl 3):S184-94.

15. Jaganath D, Mupere E. Childhood tuberculosis and malnutrition. J Infect Dis. 2012;206(12):1809-15.

16. World Health Organization. Global tuberculosis report 2018.2018 10 Dec 2018. Available from: http://apps.who.int/iris/bitstream/handle/10665/ 274453/9789241565646-eng.pdf?ua=1.

17. Adejumo OA, Daniel OJ, Adebayo Bl, Adejumo EN, Jaiyesimi EO, Akang G, et al. Treatment outcomes of childhood TB in Lagos, Nigeria. J Trop Pediatr. 2016;62(2):131-8

18. du Preez K, du Plessis L, O'Connell N, Hesseling AC. Burden, spectrum and outcomes of children with tuberculosis diagnosed at a district-level hospital in South Africa. Int J Tuberc Lung Dis. 2018;22(9):1037-43.

19. Hailu D, Abegaz WE, Belay M. Childhood tuberculosis and its treatment outcomes in Addis Ababa: a 5-years retrospective study. BMC Pediatr. 2014;14:61.

20. Tilahun G, Gebre-Selassie S. Treatment outcomes of childhood tuberculosis in Addis Ababa: a five-year retrospective analysis. BMC Public Health. 2016:16:612.

21. Enane LA, Lowenthal ED, Arscott-Mills T, Matlhare M, Smallcomb LS, Kgwaadira B, et al. Loss to follow-up among adolescents with tuberculosis in Gaborone, Botswana. Int J Tuberc Lung Dis. 2016;20(10):1320-5.

22. Mulongeni P, Hermans S, Caldwell J, Bekker LG, Wood R, Kaplan R. HIV prevalence and determinants of loss-to-follow-up in adolescents and young adults with tuberculosis in Cape Town. PLoS One. 2019;14(2):e0210937.

23. USAID. Defeat TB Annual Report October 1, 2017-September 30, 2018. 2019. Available from: https://www.urc-chs.com/sites/default/files/urc-USAIDDefeat-TB-Year1-Annual-Report.pdf.

24. Snow K, Hesseling AC, Naidoo P, Graham SM, Denholm J, du Preez K. Tuberculosis in adolescents and young adults: epidemiology and treatment outcomes in the Western cape. Int J Tuberc Lung Dis. 2017;21(6):651-7.

Ready to submit your research? Choose BMC and benefit from:

- fast, convenient online submission

- thorough peer review by experienced researchers in your field

- rapid publication on acceptance

- support for research data, including large and complex data types

- gold Open Access which fosters wider collaboration and increased citations

- maximum visibility for your research: over $100 \mathrm{M}$ website views per year

At BMC, research is always in progress.

Learn more biomedcentral.com/submissions 